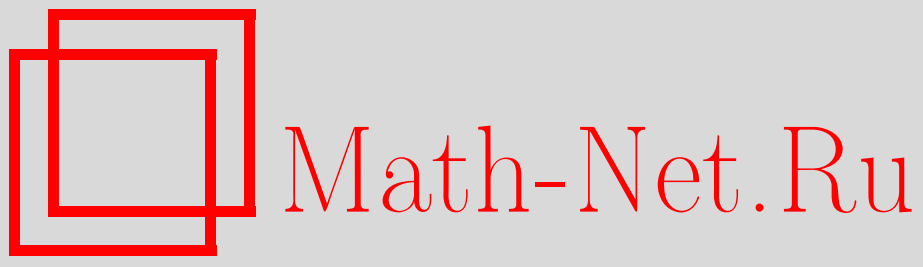

Г. В. Радзиевский, Об одном обобщении формулы Бернштейна для определения ширины полосы голоморфности функции, Функи. анализ и его прил., 1999, том 33, выпуск 2, 43-57

DOI: https://doi.org/10.4213/faa353

Использование Общероссийского математического портала MathNet.Ru подразумевает, что вы прочитали и согласны с пользовательским соглашением

http://www.mathnet.ru/rus/agreement

Параметры загрузки:

IP : 54.162 .127 .20

26 апреля 2023 г., $17: 26: 50$

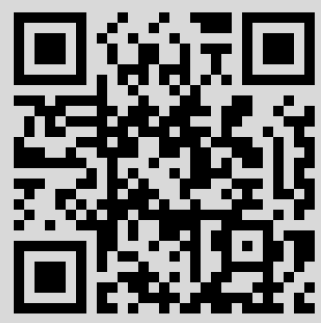


Функииональньй анализ и его приложения

1999, т. 33, вып. 2, с. 43-57

УДК $517.43+517.5$

\title{
Об одном обобщении формулы Бернштейна для определения ширины полосы голоморфности функции
}

\author{
(c) 1999. Г. В. РАдзиЕвСКий
}

\section{§1. Постановка задачи и формулировка основных результатов}

Всюду в дальнейшем $\mathbb{C}, \mathbb{R}, \mathbb{P}$ и $\mathbb{N}$ - это соответственно множества комплексных, вещественных, целых неотрицательных и целых положительных (натуральных) чисел. Пусть $B C$ - пространство непрерывных и ограниченных на $\mathbb{R}$ функций $f$ с нормой $\|f\|_{B C}=\sup _{t \in \mathbb{R}}|f(t)|$. Для каждого $\zeta>0$ введем множество $\mathfrak{G}_{\zeta}$, состоящее из целых функций $g$, удовлетворяющих условию $|g(z)| \leqslant c(g) \exp (\zeta|z|), z \in \mathbb{C}$, сужение которых на $\mathbb{R}$ принадлежит $B C$. Зададим наилучшие приближения $\mathscr{A}_{\zeta}(f)=\inf _{g \in \mathfrak{G}_{\zeta}}\|f-g\|, \zeta>0, f \in B C$. В этих обозначениях справедлива

ТЕОРЕМА БЕРнШТЕЙНА [1, работы 82 и 89]. Для того чтобы функиия $f \in$ BC допускала голоморфное продолжение в полосу $\{z \in \mathbb{C}:|\operatorname{Im} z|<H\}$, $H>0, u$ это продолжение было ограниченньм в каждой полосе $\{z \in \mathbb{C}:$ $\left.|\operatorname{Im} z| \leqslant H_{1}\right\}$ с $0<H_{1}<H$, необходимо и достаточно, итобь выполнялось неравенство

$$
\varlimsup_{\zeta \rightarrow \infty} \mathscr{A}_{\zeta}(f)^{1 / \zeta} \leqslant e^{-H}
$$

Настоящая работа посвящена распространению этой и других теорем С. Н. Бернштейна на случай аппроксимации элемента банахова пространства посредством векторов конечной степени относительно оператора, действующего в этом пространстве. Для формулировки таких обобщений понадобятся следующие понятия и определения.

Везде в этой работе $A$ - линейный, вообще говоря, неограниченный оператор, действующий в комплексном банаховом пространстве $X, I$ - тождественный оператор в $X$ и $A^{0}:=I$. Область определения, ядро и резольвентное множество оператора $A$ обозначаются соответственно через $\mathfrak{D}(A), \mathfrak{Z}(A)$ и $\rho(A)$, а $R(\lambda ; A)=(A-\lambda I)^{-1}, \lambda \in \rho(A)$, - резольвента оператора $A$. Символ $[X]$ обозначает множество ограниченных операторов, действующих в $X$, а нормы векторов и операторов обозначаются через $\|\cdot\|$, и они снабжаются индексами лишь в случаях конкретных пространств.

Элемент $g \in \mathfrak{D}_{\infty}(A):=\bigcap_{r \in \mathbb{P}} \mathfrak{D}\left(A^{r}\right)$ называется вектором степени не вылше $\zeta>0$ относительно оператора $A$, если $\left\|A^{r} g\right\| \leqslant c(g) \zeta^{r}, r \in \mathbb{P}$, с некоторой постоянной $c(g)>0$. Множество векторов степени не выше $\zeta$ относительно оператора $A$ обозначается через $\mathfrak{G}_{\zeta}(A)$, и $\mathfrak{G}_{0}(A):=\mathfrak{Z}(A)$. Очевидно, что $\mathfrak{G}_{\zeta}(A)-$ линейное многообразие в $X$ и $\mathfrak{G}_{\zeta}(A) \subseteq \mathfrak{G}_{\xi}(A)$ при $0 \leqslant \zeta<\xi$. Определим наилучшие приближения $E_{\zeta}(f, A)=\inf _{g \in \mathfrak{G}_{\zeta}(A)}\|f-g\|, \zeta \geqslant 0$, индивидуального 
элемента $f \in X$ посредством векторов степени не выше $\zeta$ относительно оператора $A$.

Элемент $f \in \mathfrak{D}_{\infty}(A)$ называется голоморфным вектором оператора $A$, если ряд

$$
e^{z A} f:=\sum_{r=0}^{\infty} \frac{z^{r} A^{r} f}{r !}
$$

сходится по норме в некоторой окрестности нуля [2]. Обозначим через $R_{A}(f)$ радиус сходимости ряда (1.2). Тогда, согласно формуле Коши-Адамара [3, теорема 3.11.4], с учетом вида ряда (1.2) и формулы Стирлинга для $r$ ! имеем

$$
R_{A}(f)=\frac{1}{e} \underset{r \rightarrow \infty}{\lim } \frac{r}{\left\|A^{r} f\right\|^{1 / r}} .
$$

Здесь предполагается, что $R_{A}(f)=\infty$, если $A^{r} f=0$ для $r \geqslant r_{0}$. Аналогичные соглашения принимаются и в других несобственных случаях и далее не оговариваются.

Пусть $\ln ^{+} a=\max \{\ln a, 0\}, a>0$, окружность радиуса $\zeta>0$ обозначим через $\mathscr{T}(\zeta)=\{\lambda \in \mathbb{C}:|\lambda|=\zeta\}$, а требование $\operatorname{mes}\{\mathscr{T}(\zeta) \cap \rho(A)\}=2 \pi \zeta$ означает, что резольвента $R\left(\zeta e^{i \theta} ; A\right)$ определена (а значит, и непрерывна) почти при всех $\theta \in[0,2 \pi]$.

Основные результаты работы установлены в предположении, что оператор принадлежит $\mathscr{R}_{\gamma}$, где через $\mathscr{R}_{\gamma}$ при $\gamma>0$ обозначено множество операторов $A$, резольвента которых удовлетворяет следующим условиям: существует такая последовательность чисел $\left\{\zeta_{m}\right\}_{m \in \mathbb{N}}$, что

$$
0<\zeta_{m} \nearrow \infty, \quad \lim _{m \rightarrow \infty} \zeta_{m+1} / \zeta_{m}=1,
$$

$\operatorname{mes}\left\{\mathscr{T}\left(\zeta_{m}\right) \cap \rho(A)\right\}=2 \pi \zeta_{m}$ и для каждого $\varepsilon>0$

$$
\lim _{m \rightarrow \infty} \int_{0}^{2 \pi} \ln ^{+}\left(e^{-\varepsilon \zeta_{m}^{\gamma}}\left\|R\left(\zeta_{m} e^{i \theta} ; A\right)\right\|\right) d \theta=0 .
$$

Теорема 1.1. Пусть $A \in \mathscr{R}_{1}$. Тогда для того итобы элемент $f$ был голоморфным вектором оператора $A$, необходимо и достаточно, чтобы $\overline{\lim }_{\zeta \rightarrow \infty} E_{\zeta}(f, A)^{1 / \zeta}<1$, в в этом случае

$$
R_{A}(f)=\varliminf_{\zeta \rightarrow \infty} \frac{1}{\zeta} \ln \frac{1}{E_{\zeta}(f, A)} .
$$

Эта теорема, как и другие теоремы, сформулированные в настоящем параграфе, будет доказана в $\S 3$. При доказательстве их используются результаты работы [4], позволяющие, в частности, получить нужную оценку сверху для $R_{A}(f)$ через $E_{\zeta}(f, A)$.

А сейчас выведем из теоремы 1.1 приведенную ранее теорему Бернштейна.

ВЫВОД ТЕОРЕМЫ БЕРНШТЕЙНА. Пусть оператор $D$ действует в пространстве $B C$ по правилу

$$
D x=-i x^{\prime}, \quad x \in \mathfrak{D}(D):=\left\{x \in B C: x^{\prime} \in B C\right\} .
$$


Резольвента оператора $D$ имеет вид

$$
\begin{array}{ll}
{[R(\lambda ; D) f](t)=i \int_{0}^{\infty} e^{i \lambda \tau} f(t-\tau) d \tau,} & \operatorname{Im} \lambda>0, \\
{[R(\lambda ; D) f](t)=i \int_{0}^{\infty} e^{-i \lambda \tau} f(t+\tau) d \tau,} & \operatorname{Im} \lambda<0 ;
\end{array}
$$

отсюда и из равенства $\left\|e^{-\eta(\cdot)}\right\|_{L_{1}[0, \infty)}=\eta^{-1}, \eta>0$, следует, что

$$
\|R(\lambda ; D)\|_{[B C]} \leqslant|\operatorname{Im} \lambda|^{-1}, \quad \lambda \in \mathbb{C}, \operatorname{Im} \lambda \neq 0 ;
$$

поэтому $D \in \mathscr{R}_{\gamma}$ при любом $\gamma>0$ и, в частности, при $\gamma=1$.

Элементарно показывается (см. вывод обобщенного неравенства Ахиезера и замечание 1 в [4]), что $\mathfrak{G}_{\zeta}(D)=\mathfrak{G}_{\zeta}$ при всех $\zeta>0$, а значит, $\mathscr{A}_{\zeta}(f)=$ $E_{\zeta}(f, D), \zeta \geqslant 0, f \in B C$.

Если $f$ голоморфна и ограничена в полосе $\left\{z \in \mathbb{C}:|\operatorname{Im} z| \leqslant H_{1}\right\}$, то

$$
f^{(r)}(t)=\frac{r !}{2 \pi i} \int_{|z-t|=H_{1}} \frac{f(z) d z}{(z-t)^{r+1}}, \quad t \in \mathbb{R} .
$$

Следовательно, $\left\|f^{(r)}\right\|_{B C} \leqslant c r ! H_{1}^{-r}$, где $c-$ верхняя грань значений функции $|f(z)|$ в полосе $\left\{z \in \mathbb{C}:|\operatorname{Im} z| \leqslant H_{1}\right\}$. Отсюда с учетом формулы (1.3) получаем

$$
H_{1} \leqslant \frac{1}{e} \varliminf_{r \rightarrow \infty} \frac{r}{\left\|f^{(r)}\right\|_{B C}^{1 / r}} \equiv \frac{1}{e} \underline{\lim _{r \rightarrow \infty}} \frac{r}{\left\|D^{r} f\right\|_{B C}^{1 / r}}=R_{D}(f) .
$$

Обратно, если $f^{(r)} \in B C, r \in \mathbb{P}$, и $R_{D}(f)>0$, то ряд

$$
\sum_{r=0}^{\infty} \frac{(i y)^{r} f^{(r)}(t)}{r !}, \quad t \in \mathbb{R}
$$

задает голоморфное продолжение $(z=t+i y)$ функции $f$ в полосу $\{z \in \mathbb{C}$ : $\left.|\operatorname{Im} z|<R_{D}(f)\right\}$ и это продолжение ограничено в каждой полосе $\{z \in \mathbb{C}:$ $\left.|\operatorname{Im} z| \leqslant H_{1}\right\}, 0<H_{1}<R_{D}(f)$. Тем самым показано, что необходимым и достаточным условием для того, чтобы функция $f$, принадлежащая $B C$, допускала голоморфное продолжение в полосу $\{z \in \mathbb{C}:|\operatorname{Im} z|<H\}, H>0$, и это продолжение было ограниченным в каждой из полос $\left\{z \in \mathbb{C}:|\operatorname{Im} z| \leqslant H_{1}\right\}$, $0<H_{1}<H$, является следующее требование: $f^{(r)} \in B C, r \in \mathbb{P}$, и $H \leqslant R_{D}(f)$. Отсюда и из теоремы 1.1 вытекает теорема Бернштейна, если заметить, что, согласно равенству $\mathscr{A}_{\zeta}(f)=E_{\zeta}(f, D)$ и формуле $(1.6)$, левая часть неравенства (1.1) совпадает с $\exp \left(-R_{D}(f)\right)$.

Вектор $f \in \mathfrak{D}_{\infty}(A)$ называется иельм вектором оператора $A$, если ряд (1.2) сходится при всех $z \in \mathbb{C}$, т. е. если $R_{A}(f)=\infty$ (см. [5]). Для целых векторов $f$ оператора $A$ приведем утверждения о вычислении порядка роста и типа целой вектор-функции $e^{z A} f$ в терминах наилучших приближений $E_{\zeta}(f, A)$. Отметим, что понятия порядка роста и типа для целых векторнозначных функций получаются видоизменением соответствующих классических понятий (см., например, [6, гл. I, §1]), в которых нужно модуль числа заменить на норму вектора [3, гл. III, §2]. В частности, формула для вычисления порядка 
роста функции, заданной рядом (1.2), принимает вид

$$
\rho:=\rho_{A}(f)=\left(1-\varlimsup_{r \rightarrow \infty} \frac{\ln \left\|A^{r} f\right\|}{r \ln r}\right)^{-1},
$$

а при $0<\rho<\infty$ формула для типа $e^{z A} f$ имеет вид

$$
\sigma:=\sigma_{A}^{(\rho)}(f)=\frac{e^{\rho-1}}{\rho} \varlimsup_{r \rightarrow \infty} \frac{\left\|A^{r} f\right\|^{\rho / r}}{r^{\rho-1}} .
$$

Для каждого вектора $f \in X$ определим число

$$
\sigma_{E}(f, A)=\inf \left\{\zeta>0: E_{\zeta}(f, A)>0\right\},
$$

считая, что $\sigma_{E}(f, A)=\infty$, когда $E_{\zeta}(f, A)>0$ для всех $\zeta>0$.

ТЕОрема 1.2. Пусть оператор А принадлежит $\mathscr{R}_{\gamma}$ при некотором $\gamma>0$. Тогда для того чтобы $f$ был иельм вектором оператора $A$ и вектор-функиия $e^{z A} f$ имела порядок роста, меньший 1 , или порядок роста, равныи $1, u$ конечный тип, необходимо и достаточно, чтобы $\sigma_{E}(f, A)<\infty$. Если $0<$ $\sigma_{E}(f, A)<\infty$, то вектор-функиия $e^{z A} f$ имеет первый порядок роста и нормальный тип.

ПРимеР 1.1. Покажем, что при $\sigma_{E}(f, A)=0$ вектор-функция $e^{z A} f$ может иметь произвольный порядок роста $\rho \in(0,1)$ и произвольный тип $\sigma \in[0, \infty]$, а также что при $\sigma_{E}(f, A)=0$ функция $e^{z A} f$ может иметь первый порядок роста и нормальный тип. Для этого в пространстве $\mathbf{m}$, состоящем из ограниченных числовых последовательностей $x=\left\{x_{j}\right\}_{j \in \mathbb{N}}$ с нормой $\|x\|_{\mathbf{m}}=\sup _{j \in \mathbb{N}}\left|x_{j}\right|$, pacсмотрим оператор $J$, действующий по правилу $J x=\left\{x_{j+1}\right\}_{j \in \mathbb{N}}$. Оператор $J$ ограничен и поэтому принадлежит $\mathscr{R}_{\gamma}$ при всех $\gamma>0$. Для него $\mathfrak{G}_{\zeta}(A)$, $0<\zeta<1$, состоит из элементов $x$, у которых $\left|x_{j}\right| \leqslant c(x) \zeta^{j-1}, j \in \mathbb{N}$, и поэтому при $0<\zeta<1$ замыкание множества $\mathfrak{G}_{\zeta}(J)$ по норме пространства $\mathbf{m}$ совпадает с подпространством $\mathbf{c}_{0}$, состоящим из сходящихся к нулю числовых последовательностей. Для каждых фиксированных $\rho \in(0,1)$ и $\sigma \in(0, \infty)$ зададим вектор $f$ последовательностью $\left\{f_{j}\right\}_{j \in \mathbb{N}}$ с $f_{1}=1$ и $f_{j+1}=\left(\rho \sigma(j / e)^{\rho-1}\right)^{j / \rho}$, $j \in \mathbb{N}$. Так как $f \in \mathbf{c}_{0}$, то $\sigma_{E}(f, J)=0$, а поскольку $\left\|J^{r} f\right\|_{\mathbf{m}}=\left(\rho \sigma(r / e)^{\rho-1}\right)^{r / \rho}$ для достаточно больших $r$, то вектор-функция $e^{z J} f$ имеет порядок роста $\rho$ и тип $\sigma$. Понятно также, как построить целый вектор $f$ оператора $J$, для которого $\sigma_{E}(f, J)=0$, а вектор-функция $e^{z J} f$ при заданном порядке роста $\rho \in(0,1)$ имеет либо минимальный, либо максимальный тип. Пусть теперь $0<\zeta<1$ и $f=\left\{\zeta^{j}\right\}_{j \in \mathbb{N}}$. Тогда $\sigma_{E}(f, J)=0$, а $e^{z J} f$ имеет порядок роста, равный 1 , и тип $\zeta$.

Зададим линейное многообразие $\mathfrak{G}_{\infty}(A)=\bigcup_{\zeta>0} \mathfrak{G}_{\zeta}(A)$, и пусть $\overline{\mathfrak{G}_{\infty}(A)}$ его замыкание по норме пространства $X$. Ясно, что $f \in \overline{\mathfrak{G}_{\infty}(A)}$ тогда и только тогда, когда $E_{\zeta}(f, A) \rightarrow 0$ при $\zeta \rightarrow \infty$. Поэтому для $f \in \overline{\mathfrak{G}_{\infty}(A)}$ корректно определена величина

$$
\beta_{A}(f):=\varlimsup_{\zeta \rightarrow \infty} \frac{\ln \zeta}{\ln \ln \frac{1}{E_{\zeta}(f, A)}},
$$

в терминах которой будет вычислен порядок роста функции $e^{z A} f$. Отметим, что пример 1.1 показывает существенность требования $\sigma_{E}(f, A)>0$, при- 
сутствующего в следующей теореме, для определения порядка роста функции $e^{z A} f$. Кроме того, формула для типа целой функции $e^{z A} f$ будет дана в предположении, что ее порядок роста больше 1. Это предположение обусловлено тем, что, согласно теореме 1.2 и примеру 1.1, при порядке роста функции $e^{z A} f$, равном 1 , можно лишь утверждать, что ее тип конечен, если $\sigma_{E}(f, A)<\infty$, и максимален, если $\sigma_{E}(f, A)=\infty$.

ТЕОрема 1.3. Пусть $A \in \mathscr{R}_{\gamma}$ при некотором $\gamma \geqslant 1, a f-$ такой вектор, ито $\sigma_{E}(f, A)>0$. Тогда для того чтобы $f$ был иельм вектором оператора A и вектор-функиия $e^{z A} f$ имела конечный порядок роста $\rho<\gamma(\gamma-1)^{-1}$, необходимо и достаточно, чтобь $f \in \overline{\mathfrak{G}_{\infty}(A)}$ u $\beta_{A}(f)<\gamma^{-1}$. Eсли $f \in \overline{\mathfrak{G}_{\infty}(A)}$ u $\beta_{A}(f)<\gamma^{-1}$, то $\rho=\left(1-\beta_{A}(f)\right)^{-1}$, а в случае $\rho>1$ mип $\sigma$ функиии $e^{z A} f$ вьчисляется по формуле

$$
\sigma=\frac{1}{\rho-1}\left(\frac{\rho-1}{\rho}\right)^{\rho}\left(\varlimsup_{\zeta \rightarrow \infty} \frac{\zeta^{\rho /(\rho-1)}}{-\ln E_{\zeta}(f, A)}\right)^{\rho-1} .
$$

При дополнительных требованиях относительно оператора $A$ можно также указать формулу для вычисления типа функции $e^{z A} f$ в терминах $E_{\zeta}(f, A)$ в случае, когда порядок ее роста равен 1. Одним из таких требований (важным в приложениях) является предположение о мероморфности резольвенты оператора $A$, означающее, что весь спектр оператора $A$ состоит лишь из изолированных собственных значений $\lambda_{k}(A)$, которые являются полюсами резольвенты $R(\lambda ; A)$. Из мероморфности резольвенты оператора $A$ следует, что длина каждой цепочки собственного и присоединенных к нему векторов, отвечающей $\lambda_{k}(A)$, конечна и не превосходит порядка полюса резольвенты $R(\lambda ; A)$ в точке $\lambda_{k}(A)$. При этом кратность самого собственного значения $\lambda_{k}(A)$ может оказаться равной и бесконечности, если бесконечным окажется число линейно независимых собственных векторов, отвечающих $\lambda_{k}(A)$.

Теорема 1.4. Пусть оператор А имеет мероморфную резольвенту. Тогда $f$ - цельй вектор оператора $A$ и вектор-функиия $e^{z A} f$ имеет первый порядок роста и нормальный тип $\sigma$ в том и только том случае, когда $0<\sigma_{E}(f, A)<\infty$. Если $0<\sigma_{E}(f, A)<\infty$, mo $\sigma=\sigma_{E}(f, A)$. Чuсло $\sigma_{E}(f, A)$ равно 0 в том и только том случае, когда либо $f=0$, либо $e^{z A} f=\sum_{s=0}^{d}(s !)^{-1} z^{s} y_{d-s}$, где $y_{0}, \ldots, y_{d}$ - иепочка собственного и присоединенных $\kappa$ нему векторов оператора $A$, отвечаюшая нулевому собственному значению $\left(m . e . f=y_{d}\right)$.

Теорема 1.4 установлена в $\S 3$ без предположения о том, что $A \in \mathscr{R}_{\gamma}$. При доказательстве ее используется следуюшее утверждение, которое дает описание многообразия $\mathfrak{G}_{\zeta}(A)$.

ПРЕДЛОЖЕНИЕ 1.1. Пусть оператор А имеет мероморфную резольвенту, a $\mathfrak{N}_{k}$ и $\mathfrak{P}_{k}$ - множества, состоящие соответственно из собственньх $и$ собственных и присоединенных $\kappa$ ним векторов оператора $A$, отвечающих собственному значению $\lambda_{k}(A)$, с добавленным $\kappa$ ним нулевым вектором. Тогда $\mathfrak{N}_{k}$ и $\mathfrak{P}_{k}$ - подпространства, сумма любого конечного числа подпространств $\mathfrak{P}_{k}$ при различных $k$ являетсл прямой $и$

$$
\left.\mathfrak{G}_{\zeta}(A)=\left(\underset{k:\left|\lambda_{k}(A)\right|=\zeta}{\dot{+}} \mathfrak{N}_{k}\right) \dot{+} \underset{k:\left|\lambda_{k}(A)\right|<\zeta}{\dot{+}} \mathfrak{P}_{k}\right) .
$$


Здесь через $\dot{+}$ обозначена прямая сумма соответствуюших подпространств $u \mathfrak{G}_{\zeta}(A)=\{0\}$, если $\zeta<\left|\lambda_{k}(A)\right|$ для всех $k$.

Это утверждение установлено при доказательстве леммы 1 работы [4] (см. формулу (30) и следующие за ней рассуждения в [4]).

Приведем следствие из теоремы 1.1 , относяшееся к решениям $x$ уравнения в свертке (см., например, $[6,7])$

$$
\int_{-\pi}^{\pi} x(t+z) d \mu(t)=0
$$

в предположении, что функция $\mu$ имеет ограниченную вариацию на $[-\pi, \pi]$, а $\pi$ и $-\pi$ - ее точки роста. Пусть $\lambda_{k}$ - нули целой функции

$$
\Delta(\lambda)=\int_{-\pi}^{\pi} e^{i \lambda t} d \mu(t)
$$

a $d_{k}$ - их кратности. Легко показать, что у функции $\Delta$ бесконечное число нулей и, как следует из доказательства теоремы 1.4.1 монографии [7], существует такая последовательность $\left\{\zeta_{m}\right\}_{m \in \mathbb{N}}$, удовлетворяющая соотношениям $(1.4)$, что для каждого $\varepsilon>0$ найдется постоянная $c_{\varepsilon}$, для которой

$$
\ln \left|\Delta\left(\zeta_{m} e^{i \theta}\right)\right| \geqslant(\pi|\sin \theta|-\varepsilon) \zeta_{m}+c_{\varepsilon}, \quad m \in \mathbb{N}, 0 \leqslant \theta<2 \pi .
$$

Пусть функции $x_{k, l}$ заданы формулами

$$
x_{k, l}(t)=(l !)^{-1}(i t)^{l} e^{i \lambda_{k} t}, \quad l=0, \ldots, d_{k-1},
$$

а наилучшие приближения по этим функциям - формулами

$$
\mathscr{E}_{\zeta}(f)_{\mu}=\inf _{\alpha_{k}, l \in \mathbb{C}}\left\|f-\sum_{k:\left|\lambda_{k}\right| \leqslant \zeta} \sum_{l=0}^{d_{k}-1} \alpha_{k, l} x_{k, l}\right\|_{C}, \quad \zeta>0, f \in C,
$$

где $C$ - пространство непрерывных на $[-\pi, \pi]$ функций.

Во введенных обозначениях и предположениях справедливо

СлЕДСТВИЕ 1.1. Для того чтобы существовало голоморфное в области $Q(H)=\bigcup_{-\pi \leqslant t \leqslant \pi}\{z \in \mathbb{C}:|z-t|<H\}, H>0$, решение $x$ уравнения (1.12), удовлетворяющее условию $x(t)=f(t),-\pi \leqslant t \leqslant \pi$, где $f \in C$, необходимо и достаточно, чтобь

$$
\varlimsup_{\zeta \rightarrow \infty} \mathscr{E}_{\zeta}(f)_{\mu}^{1 / \zeta} \leqslant e^{-H}
$$

ДоказАтЕльство. Введем функционал $U(x)=\int_{-\pi}^{\pi} x(t) d \mu(t), x \in C$, и оператор $D_{\mu}$, действующий в пространстве $C$ по правилу

$$
D_{\mu} x=-i x^{\prime}, \quad x \in \mathfrak{D}\left(D_{\mu}\right):=\left\{x \in C^{1}[-\pi, \pi]: U(x)=0\right\} .
$$


Резольвента оператора $D_{\mu}$ представима в виде

$$
\begin{aligned}
& {\left[R\left(\lambda ; D_{\mu}\right) f\right](t)=i \int_{-\pi}^{t} e^{i \lambda(t-\tau)} f(\tau) d \tau-\frac{i e^{i \lambda t}}{\Delta(\lambda)} \int_{-\pi}^{\pi}\left(\int_{-\pi}^{\tau} e^{i \lambda(\tau-\xi)} f(\xi) d \xi\right) d \mu(\tau),} \\
& {\left[R\left(\lambda ; D_{\mu}\right) f\right](t)=-i \int_{t}^{\pi} e^{i \lambda(t-\tau)} f(\tau) d \tau+\frac{i e^{i \lambda t}}{\Delta(\lambda)} \int_{-\pi}^{\pi}\left(\int_{\tau}^{\pi} e^{i \lambda(\tau-\xi)} f(\xi) d \xi\right) d \mu(\tau) .}
\end{aligned}
$$

При $\lambda \neq \lambda_{k}$ оба эти представления совпадают, однако равенство (1.15) используется для оценки нормы резольвенты $R\left(\lambda ; D_{\mu}\right)$ при $\operatorname{Im} \lambda \geqslant 0$, a $(1.16)$ 一 при $\operatorname{Im} \lambda \leqslant 0$. Из (1.13), (1.15) и (1.16) выводим, что $\left\|R\left(\lambda ; D_{\mu}\right)\right\|_{[C]} \leqslant$ $c_{\varepsilon}^{\prime} \exp (\varepsilon|\lambda|)$, где $|\lambda|=\zeta_{m}$, постоянная $c_{\varepsilon}^{\prime}>0$ не зависит от $m \in \mathbb{N}$, а последовательность $\left\{\zeta_{m}\right\}_{m \in \mathbb{N}}$ удовлетворяет соотношениям (1.4). Таким образом, оператор $D_{\mu}$ принадлежит $\mathscr{R}_{1}$ и к нему применима теорема 1.1 .

Из (1.15) или (1.16) следует мероморфность резольвенты оператора $D_{\mu}$, а в силу определения этого оператора его собственные значения $\lambda_{k}\left(D_{\mu}\right)$ совпадают с нулями $\lambda_{k}$ целой функции $\Delta$ и каждому $\lambda_{k}\left(D_{\mu}\right)$ отвечает одна цепочка собственной и присоединенных к ней функций $x_{k, 0}, \ldots, x_{k, d_{k}-1}$. Отсюда и из предложения 1.1 заключаем, что

$$
\mathscr{E}_{\zeta}(f)_{\mu}=\lim _{\xi \rightarrow \zeta+0} E_{\xi}\left(f, D_{\mu}\right)
$$

Далее, как и при выводе теоремы Бернштейна, показывается, что необходимым и достаточным условием для того, чтобы у уравнения (1.12) существовало голоморфное в области $Q(H)$ решение, удовлетворяюшее условию $x(t)=f(t),-\pi \leqslant t \leqslant \pi$, является следующее требование: $f$ бесконечно дифференцируема, $U\left(f^{(r)}\right)=0, r \in \mathbb{P}\left(\right.$ т. е. $\left.f \in \mathfrak{D}_{\infty}\left(D_{\mu}\right)\right)$, и $H \leqslant R_{D_{\mu}}(f)$. Отсюда и из теоремы 1.1 вытекает утверждение следствия 1.1, если заметить, что, согласно равенствам (1.6) и (1.17), левая часть неравенства (1.14) совпадает с $\exp \left(-R_{D_{\mu}}(f)\right)$.

Поскольку оператор $D_{\mu}$ принадлежит $\mathscr{R}_{1}$ и имеет мероморфную резольвенту, то понятно, как формулируются и выводятся из теорем 1.3 и 1.4 следствия о вычислении в терминах $\mathscr{E}_{\zeta}(f)_{\mu}$ порядка роста и типа целого решения $x$ уравнения (1.12), удовлетворяющего условию $x(t)=f(t),-\pi \leqslant t \leqslant \pi$. Отметим также, что классы операторов $\mathscr{R}_{\gamma}$ достаточно широки и им, в частности, при любом $\gamma>0$ принадлежат операторы, порождаемые нелокальными регулярными в смысле [8] краевыми задачами для обыкновенных функциональнодифференциальных выражений.

\section{§ 2. Вспомогательные утверждения}

Далее $L_{1}$ - пространство суммируемых на $[0,2 \pi]$ функций с нормой $\|\cdot\|_{1}$. Через $H_{1}$ обозначим класс Харди голоморфных в единичном круге функций, для которых $\left\|\alpha\left(\zeta e^{i(\cdot)}\right)\right\|_{1}$ равномерно ограничена по $\zeta \in(0,1)$. Если $\alpha \in H_{1}$, то для почти всех $\theta \in[0,2 \pi]$ существует радиальный предел $\lim _{\zeta \rightarrow 1-0} \alpha\left(\zeta e^{i \theta}\right)=$ : $\alpha\left(e^{i \theta}\right)$ и $\alpha\left(e^{i(\cdot)}\right) \in L_{1}$. 
Пусть $\varphi$ - измеримая на $[0,2 \pi]$ функция и $\varphi(\theta) \geqslant 0$ почти при всех $\theta \in$ $[0,2 \pi]$. Определим классы функций

$$
\Phi_{1}(\varphi)=\left\{\alpha \in H_{1}: \alpha(0)=1, \alpha\left(e^{i(\cdot)}\right) \varphi(\cdot) \in L_{1}\right\},
$$

и

$$
\Phi_{r}(\varphi)=\left\{\alpha \in \Phi_{1}(\varphi): \alpha^{\prime}(0)=\cdots=\alpha^{(r-1)}(0)=0\right\}
$$

для натуральных чисел $r \geqslant 2$. Зададим числа

$$
\nu_{r}(\varphi)=\frac{1}{2 \pi} \inf _{\alpha \in \Phi_{r}(\varphi)} \int_{0}^{2 \pi}\left|\alpha\left(e^{i \theta}\right)\right| \varphi(\theta) d \theta,
$$

причем если множество $\Phi_{r}(\varphi)$ пусто, то считаем, что $\nu_{r}(\varphi)=\infty$.

ПрЕДЛОЖЕНИЕ 2.1. Пусть $\operatorname{mes}\{\mathscr{T}(\zeta) \cap \rho(A)\}=2 \pi \zeta$ для некоторого $\zeta>0$. Тогда для любого $r \in \mathbb{N}$ справедливы оценки

$$
\begin{aligned}
E_{\zeta}(f, A) & \leqslant \zeta^{-r+1} \nu_{r}\left(\left\|R\left(\zeta e^{i(\cdot)} ; A\right)\right\|\right)\left\|A^{r} f\right\|, & & f \in \mathfrak{D}\left(A^{r}\right), \\
\left\|A^{r} g\right\| & \leqslant \zeta^{r+1} \nu_{r+1}\left(\left\|R\left(\zeta e^{i(\cdot)} ; A\right)\right\|\right)\|g\|, & & g \in \mathfrak{G}_{\zeta}(A) .
\end{aligned}
$$

ДокАзАТЕльство. Оценка (2.1) установлена в работе [4] и является непосредственным следствием определения функции Фавара, введенной в [4] равенством (37), и отмеченного там же неравенства (38).

Установим $(2.2)$ в предположении конечности числа $\nu_{r+1}\left(\left\|R\left(\zeta e^{i(\cdot)} ; A\right)\right\|\right)$, т. е. в предположении, что множество $\Phi_{r+1}\left(\left\|R\left(\zeta e^{i(\cdot)} ; A\right)\right\|\right)$ непусто (в противном случае это неравенство очевидно). Пусть $\alpha-$ произвольная функция из $\Phi_{r+1}\left(\left\|R\left(\zeta e^{i(\cdot)} ; A\right)\right\|\right)$. Из условия $\operatorname{mes}\{\mathscr{T}(\zeta) \cap \rho(A)\}=2 \pi \zeta$ следует непрерывность почти всюду функции $R\left(\zeta e^{i(\cdot)} ; A\right)$ по операторной норме, а так как $\alpha \in H_{1}$ и $\int_{0}^{2 \pi}\left|\alpha\left(e^{i \theta}\right)\right|\left\|R\left(\zeta e^{i \theta} ; A\right)\right\| d \theta<\infty$, то из [3, теоремы 3.5.3 и 3.7.4] вытекает интегрируемость в смысле Бохнера на $[0,2 \pi]$ операторнозначной функции $\overline{\alpha(\cdot)} R\left(\zeta e^{i(\cdot)} ; A\right)$. Значит, определены и являются ограниченными операторы

$$
B_{k}:=\frac{1}{2 \pi} \int_{0}^{2 \pi} e^{-i k \theta} \overline{\alpha\left(e^{-i \theta}\right)} R\left(\zeta e^{-i \theta} ; A\right) d \theta .
$$

Из тождества $R(\lambda ; A) A g=g+\lambda R(\lambda ; A) g, \lambda \in \rho(A), g \in \mathfrak{D}(A)$, и из предположения, что $\operatorname{mes}\{\mathscr{T}(\zeta) \cap \rho(A)\}=2 \pi \zeta$, имеем

$$
B_{k} A g=\frac{1}{2 \pi}\left(\int_{0}^{2 \pi} e^{-i k \theta} \overline{\alpha\left(e^{-i \theta}\right)} d \theta\right) g+\zeta B_{k+1} g, \quad k=0, \pm 1, \ldots, g \in \mathfrak{D}(A),
$$

а так как $\alpha \in H_{1}$, то интеграл в правой части этого равенства равен нулю, когда $k=-1,-2, \ldots$ Поэтому для векторов $g \in \mathfrak{G}_{\zeta}(A)$ справедливы тождества $B_{0} g=\zeta^{-k} B_{-k} A^{k} g, k \in \mathbb{N}$. Так как $g \in \mathfrak{G}_{\zeta}(A)$, то $\sup _{k \in \mathbb{N}} \zeta^{-k}\left\|A^{k} g\right\|<\infty$, а учитывая, что $\left\|B_{-k}\right\| \rightarrow 0$ при $k \rightarrow \infty$ (это доказывается точно так же, как и известный факт о стремлении к нулю коэффициентов Фурье числовой суммируемой функции), получаем равенство $B_{0} g=0$ для всех $g \in \mathfrak{G}_{\zeta}(A)$.

Функция $\alpha$ принадлежит $H_{1}$ и поэтому интеграл в правой части равенства (2.3) равен $(k !)^{-1} \overline{\alpha^{(k)}(0)}$ при $k \in \mathbb{P}$, а так как $\alpha \in \Phi_{r+1}(\varphi)$, то $\alpha(0)=1$ и 
$\alpha^{\prime}(0)=\cdots=\alpha^{(r)}(0)=0$. Отсюда и из равенства $B_{0} g=0$ для $g \in \mathfrak{G}_{\zeta}(A)$ выводим тождества $g+\zeta B_{1} g=0, B_{k} A g=\zeta B_{k+1} g, k=1, \ldots, r$. Из этих тождеств с учетом того, что $A g \in \mathfrak{G}_{\zeta}(A)$, если $g \in \mathfrak{G}_{\zeta}(A)$, следует, что $A^{r} g=-\zeta^{r+1} B_{r+1} g$. Воспользовавшись теперь определением операторов $B_{k}$, получаем, что

$$
\left\|A^{r} g\right\| \leqslant \frac{\zeta^{r+1}}{2 \pi}\left(\int_{0}^{2 \pi}\left|\alpha\left(e^{i \theta}\right)\right|\left\|R\left(\zeta e^{i \theta} ; A\right)\right\| d \theta\right)\|g\| .
$$

Поскольку $\alpha$ - произвольная функция из $\Phi_{r+1}\left(\left\|R\left(\zeta e^{i(\cdot)} ; A\right)\right\|\right)$, то из этого неравенства и из определения числа $\nu_{r}(\varphi)$ вытекает оценка $(2.2)$.

В $[4$, лемма 3$]$ установлена следующая оценка для $\nu_{r}(\varphi)$.

ПрЕДЛОЖЕНИЕ 2.2. Пусть $\varphi(\theta) \geqslant 0$ почти при всех $\theta \in[0,2 \pi]$. Если $1 / \varphi \in$ $L_{1} \quad u \int_{0}^{2 \pi} \ln ^{+} \varphi(\theta) d \theta<\infty, m o$

$$
\nu_{r}(\varphi) \leqslant \exp \left(\frac{2 r-1}{2 \pi} \int_{0}^{2 \pi} \ln ^{+} \varphi(\theta) d \theta\right), \quad r \in \mathbb{N} .
$$

Основной в этом параграфе является

Лемма 2.1. Пусть $A \in \mathscr{R}_{\gamma}$ при некотором $\gamma>0$. Тогда для любого $\varepsilon>0$ найдутся такие положительнье постоянные $c_{\varepsilon}, \zeta_{\varepsilon} u \eta_{\varepsilon}$, ито

1) для каждого $r \in \mathbb{N}$ и каждого $f \in \mathfrak{D}\left(A^{r}\right)$ выполнена оченка

$$
E_{\zeta}(f, A) \leqslant e^{\varepsilon\left(r+\zeta^{\gamma}\right)} \zeta^{-r}\left\|A^{r} f\right\|, \quad \zeta \geqslant \zeta_{\varepsilon} ;
$$

2) если при некотором $\varepsilon>0$

$$
\int_{0}^{\infty} e^{2 \varepsilon \xi^{\gamma}} E_{\xi}(f, A) d \xi<\infty,
$$

то $f \in \mathfrak{D}_{\infty}(A)$ и для всех $r \in \mathbb{N}$ выполнена оченка

$$
\left\|A^{r} f\right\| \leqslant c_{\varepsilon} e^{\varepsilon r}\left\{\eta_{\varepsilon}^{r}\|f\|+\int_{0}^{\infty} e^{\varepsilon \xi^{\gamma}} \xi^{r-1} E_{\xi}(f, A) d \xi\right\} .
$$

Доказательство. Так как $A \in \mathscr{R}_{\gamma}$, то из (1.4) и (1.5) следует существование $m_{1} \in \mathbb{N}$, для которого

$$
\begin{aligned}
& \zeta_{m+1} / \zeta_{m} \leqslant 1+\varepsilon, \quad m \geqslant m_{1}, \\
& \frac{1}{\pi} \int_{0}^{2 \pi} \ln ^{+}\left(e^{-\varepsilon \zeta_{m}^{\gamma}} \zeta_{m}\left\|R\left(\zeta_{m} e^{i \theta} ; A\right)\right\|\right) d \theta \leqslant \varepsilon, \quad m \geqslant m_{1} .
\end{aligned}
$$

Из условия $\operatorname{mes}\left\{\mathscr{T}\left(\zeta_{m}\right) \cap \rho(A)\right\}=2 \pi \zeta_{m}$ и резольвентного тождества выводится ограниченность функции $\left\|R\left(\zeta_{m} e^{i(\cdot)} ; A\right)\right\|^{-1}$. Таким образом, функция $\varphi(\cdot)=e^{-\varepsilon \zeta_{m}^{\gamma}} \zeta_{m}\left\|R\left(\zeta_{m} e^{i(\cdot)} ; A\right)\right\|$ удовлетворяет требованиям предложения 2.2, и из него и из оценки (2.8) следует, что

$$
\nu_{r}\left(e^{-\varepsilon \zeta_{m}^{\gamma}} \zeta_{m}\left\|R\left(\zeta_{m} e^{i(\cdot)} ; A\right)\right\|\right) \leqslant e^{\varepsilon r}, \quad m \geqslant m_{1} .
$$

Так как $\nu_{r}(\mu \varphi)=\mu \nu_{r}(\varphi)$, если постоянная $\mu$ положительна, то из (2.1) и (2.9) получаем

$$
E_{\zeta_{m}}(f, A) \leqslant e^{\varepsilon\left(r+\zeta_{m}^{\gamma}\right)} \zeta_{m}^{-r}\left\|A^{r} f\right\|, \quad f \in \mathfrak{D}\left(A^{r}\right), m \geqslant m_{1} .
$$


Последовательность $\left\{\zeta_{m}\right\}_{m \in \mathbb{N}}$ возрастает и стремится к бесконечности, и поэтому для каждого $\zeta \geqslant \zeta_{m_{1}}$ найдется такое натуральное число $m \geqslant m_{1}$, для которого $\zeta_{m} \leqslant \zeta<\zeta_{m+1}$. Отсюда и из неравенства (2.7) следует, что $\zeta / \zeta_{m} \leqslant 1+\varepsilon \leqslant e^{\varepsilon}$. Учитывая теперь, что $E_{\zeta}(f, A)$ не возрастает по $\zeta$, в силу неравенства (2.10) получим

$$
E_{\zeta}(f, A) \leqslant E_{\zeta_{m}}(f, A) \leqslant e^{\varepsilon\left(2 r+\zeta^{\gamma}\right)} \zeta^{-r}\left\|A^{r} f\right\|, \quad \zeta \geqslant \zeta_{m_{1}} .
$$

Заменив в этих неравенствах $\varepsilon$ на $\varepsilon / 2$, придем к (2.4).

Для доказательства оценки (2.6) покажем сначала существование такой подпоследовательности $\left\{\zeta_{m_{k}}\right\}_{k \in \mathbb{N}}$ последовательности $\left\{\zeta_{m}\right\}_{m \in \mathbb{N}}$, что

$$
1+\varepsilon \leqslant \zeta_{m_{k+1}} / \zeta_{m_{k}}<(1+\varepsilon)^{2}, \quad k \in \mathbb{N} .
$$

Считая $m_{1}$ таким же, как и в $(2.7)$, покажем, как по $m_{k}$ находится $m_{k+1}$. Если $\zeta_{m_{k}+1} / \zeta_{m_{k}}=1+\varepsilon$, то $m_{k+1}=m_{k}+1$. Пусть теперь $\zeta_{m_{k}+1} / \zeta_{m_{k}}<1+\varepsilon$, а $j$ - наибольшее натуральное число, для которого $\zeta_{m_{k}+j} / \zeta_{m_{k}}<1+\varepsilon$. Отсюда и из неравенства (2.7) имеем

$$
1+\varepsilon \leqslant \zeta_{m_{k}+j+1} / \zeta_{k}=\left(\zeta_{m_{k}+j+1} / \zeta_{m_{k}+j}\right)\left(\zeta_{m_{k}+j} / \zeta_{m_{k}}\right)<(1+\varepsilon)^{2} .
$$

Значит, положив $m_{k+1}=m_{k}+j+1$, получим (2.11). Обозначим для краткости $\zeta_{m_{k}}$ через $\eta_{k}$, и тогда неравенства $(2.11)$ запишутся в виде

$$
1+\varepsilon \leqslant \eta_{m+1} / \eta_{m}<(1+\varepsilon)^{2}, \quad m \in \mathbb{N} .
$$

Поскольку $\left\{\eta_{m}\right\}_{m \in \mathbb{N}}$ - подпоследовательность последовательности $\left\{\zeta_{m}\right\}_{m \in \mathbb{N}}$, то неравенство (2.9) справедливо, если в нем $\zeta_{m}$ заменить на $\eta_{m}$. Отсюда, из тождества $\nu_{r}(\mu \varphi)=\mu \nu_{r}(\varphi), \mu>0$, и из оценки $(2.2)$ заключаем, что

$$
\left\|A^{r} g\right\| \leqslant e^{\varepsilon\left(2 r+\eta_{m}^{\gamma}\right)} \eta_{m}^{r}\|g\|, \quad g \in \mathfrak{G}_{\eta_{m}}(A) .
$$

Покажем теперь справедливость неравенства

$$
e^{\varepsilon\left(2 r+\eta_{m+2}^{\gamma}\right)} \eta_{m+2}^{r} E_{\eta_{m+1}}(f, A) \leqslant c_{\varepsilon}^{\prime} e^{6 \varepsilon r} \int_{\eta_{m}}^{\eta_{m+1}} e^{6 \varepsilon \xi^{\gamma}} \xi^{r-1} E_{\xi}(f, A) d \xi, \quad m, r \in \mathbb{N},
$$

в предположении, что $(1+\varepsilon)^{4 \gamma} \leqslant 6$. Из этого предположения и из оценок $(2.12)$ имеем $6 \eta_{m}^{\gamma} \geqslant \eta_{m+2}^{\gamma}$ и $\eta_{m} / \eta_{m+2} \geqslant(1+\varepsilon)^{-4} \geqslant e^{-4 \varepsilon}$. Поэтому

$$
\begin{aligned}
\int_{\eta_{m}}^{\eta_{m+1}} e^{6 \varepsilon \xi^{\gamma}} \xi^{r-1} E_{\xi}(f, A) d \xi & \geqslant e^{6 \varepsilon \eta_{m}^{\gamma}} \eta_{m}^{r} E_{\eta_{m+1}}(f, A) \ln \frac{\eta_{m+1}}{\eta_{m}} \\
& \geqslant e^{\varepsilon \eta_{m+2}^{\gamma}} e^{-4 r \varepsilon} \eta_{m+2}^{r} E_{\eta_{m+1}}(f, A) \ln (1+\varepsilon),
\end{aligned}
$$

откуда следует (2.14).

Понятно, что утверждение 2 достаточно установить лишь для малых положительных $\varepsilon$. Поэтому далее считаем, что $(1+\varepsilon)^{4 \gamma} \leqslant 6$, и тогда выполнена оценка (2.14). Кроме того, не уменьшая общности, предположим, что вместо условия (2.5) справедливо требование

$$
\int_{0}^{\infty} e^{12 \varepsilon \xi^{\gamma}} E_{\xi}(f, A) d \xi<\infty
$$

(В дальнейшем из этого требования после замены $\varepsilon$ на $\varepsilon / 6$ получается доказываемая оценка (2.6).) 
Зафиксируем $\delta>0$ и для вектора $f$ найдем такие элементы $g_{\delta, r, m} \in \mathfrak{G}_{\eta_{m}}(A)$, что

$$
\left\|f-g_{\delta, r, m}\right\| \leqslant E_{\eta_{m}}(f, A)+\delta 2^{-m} e^{-\varepsilon \eta_{m+1}^{\gamma}} \eta_{m+1}^{-r}, \quad m, r \in \mathbb{N} .
$$

Следовательно,

$$
\left\|g_{\delta, r, m+1}-g_{\delta, r, m}\right\| \leqslant 2 E_{\eta_{m}}(f, A)+\delta 2^{-m+1} e^{-\varepsilon \eta_{m+1}^{\gamma}} \eta_{m+1}^{-r},
$$

а так как $g_{\delta, r, m} \in \mathfrak{G}_{\eta_{m}}(A)$, то $g_{\delta, r, m+1}-g_{\delta, r, m} \in \mathfrak{G}_{\eta_{m+1}}$, и поэтому из $(2.13)$ заключаем, что

$$
\begin{aligned}
\left\|A^{r}\left(g_{\delta, r, m+1}-g_{\delta, r, m}\right)\right\| & \leqslant e^{\varepsilon\left(2 r+\eta_{m+1}^{\gamma}\right)} \eta_{m+1}^{r}\left\|g_{\delta, r, m+1}-g_{\delta, r, m}\right\| \\
& \leqslant 2 e^{\varepsilon\left(2 r+\eta_{m+1}^{\gamma}\right)} \eta_{m+1}^{r} E_{\eta_{m}}(f, A)+\delta 2^{-m+1} e^{2 \varepsilon r} .
\end{aligned}
$$

Отсюда и из (2.14) получаем

$$
\sum_{m=2}^{\infty}\left\|A^{r}\left(g_{\delta, r, m+1}-g_{\delta, r, m}\right)\right\| \leqslant 2 c_{\varepsilon}^{\prime} e^{6 \varepsilon r} \int_{0}^{\infty} e^{6 \varepsilon \xi^{\gamma} \xi^{r-1}} E_{\xi}(f, A) d \xi+\delta e^{2 \varepsilon r}, \quad r \in \mathbb{N} .
$$

Поскольку вектор $f$ удовлетворяет требованию (2.15), то из оценки (2.17) следует фундаментальность последовательностей векторов $\left\{A^{r} g_{\delta, r, m}\right\}_{m \in \mathbb{N}}$ при каждом $r$. Обозначим через $y_{r}$ предельные элементы этих последовательностей, и тогда

$$
y_{r}=A^{r} g_{\delta, r, 2}+\sum_{m=2}^{\infty} A^{r}\left(g_{\delta, r, m+1}-g_{\delta, r, m}\right), \quad r \in \mathbb{N} .
$$

Из (2.15) следует, что $E_{\xi}(f, A) \rightarrow 0$, когда $\xi \rightarrow \infty$. Поэтому из (2.16) вытекает сходимость при всех $r \in \mathbb{N}$ последовательностей векторов $\left\{g_{\delta, r, m}\right\}_{m \in \mathbb{N}}$ к вектору $f$. А так как резольвентное множество оператора $A$ непусто, то операторы $A^{r}$ замкнуты $\left[9\right.$, гл. VII, §9]. Значит, $f \in \mathfrak{D}\left(A^{r}\right)$ и $A^{r} f=y_{r}, r \in \mathbb{N}$, т. е. $f \in \mathfrak{D}_{\infty}(A)$.

Оценим теперь $\left\|A^{r} f\right\|$. Так как $E_{\xi}(f, A) \leqslant\|f\|$, то из (2.16) следует неравенство

$$
\left\|g_{\delta, r, 2}\right\| \leqslant 2\|f\|+\delta 2^{-2} e^{-\varepsilon \eta_{3}^{\gamma}} \eta_{3}^{-r}
$$

а поскольку $g_{\delta, r, 2} \in \mathfrak{G}_{\eta_{2}}(A)$, то из (2.13) выводим оценку

$$
\left\|A^{r} g_{\delta, r, 2}\right\| \leqslant 2 e^{\varepsilon\left(2 r+\eta_{2}^{\gamma}\right)} \eta_{2}^{r}\|f\|+\delta 2^{-2} e^{2 \varepsilon r}, \quad r \in \mathbb{N} .
$$

Из нее и неравенства (2.17) с учетом равенства (2.18), в котором, как было показано, $y_{r}=A^{r} f$, заключаем, что

$$
\left\|A^{r} f\right\| \leqslant 2 \max \left\{e^{\varepsilon \eta_{2}^{\gamma}}, c_{\varepsilon}^{\prime}\right\} e^{6 \varepsilon r}\left\{\eta_{2}^{r}\|f\|+\int_{0}^{\infty} e^{6 \varepsilon \xi^{\gamma} \xi^{r-1}} E_{\xi}(f, A) d \xi+\delta\right\}, \quad r \in \mathbb{N} .
$$

Левая часть этого неравенства не зависит от $\delta$, а в правой $\delta$ произвольное число, причем $\varepsilon, c_{\varepsilon}^{\prime}$ и $\eta_{2}$ не зависят от $\delta$. Поэтому неравенство (2.19) справедливо при $\delta=0$, и тогда из него после замены $\varepsilon$ на $\varepsilon / 6$ получается оценка (2.6). Отметим, что при этой замене требование (2.15) записывается в виде (2.5). Тем самым доказано второе утверждение леммы. 


\section{§3. Доказательство основных результатов}

Теоремы 1.1 и 1.3 будут выведены из следуюшего утверждения.

Теорема 3.1. Пусть $A \in \mathscr{R}_{\gamma}$ при некотором $\gamma>0$. Тогда для того итобы вектор $f$ принадлежал $\mathfrak{D}_{\infty}(A)$ и число

$$
a_{\gamma}:=\varlimsup_{r \rightarrow \infty} \frac{\left\|A^{r} f\right\|^{\gamma / r}}{r}
$$

было конечныл, необходимо и достаточно, итобь $f$ принадлежал $\overline{\mathfrak{G}_{\infty}(A)}$ u конечным было число

$$
b_{\gamma}:=\varlimsup_{\zeta \rightarrow \infty} \frac{\zeta^{\gamma}}{-\ln E_{\zeta}(f, A)} .
$$

Числа $a_{\gamma}$ и $b_{\gamma}$ связаны равенством еү $a_{\gamma}=b_{\gamma}$.

ДокАЗАТЕльство. Пусть $f \in \mathfrak{D}_{\infty}(A)$ и $a_{\gamma}<\infty$. Тогда для произвольного $\delta>0$ найдется натуральное число $r_{\delta}$, для которого

$$
\frac{\left\|A^{r} f\right\|^{\gamma / r}}{r} \leqslant a_{\gamma}+\delta, \quad r \geqslant r_{\delta} .
$$

Далее считаем, что $\varepsilon$ удовлетворяет неравенствам

$$
0<\varepsilon<\gamma^{-1}\left(1+e\left(a_{\gamma}+\delta\right)\right)^{-1} .
$$

Воспользовавшись первым утверждением леммы 2.1 и оценкой $(3.3)$, получим

$$
E_{\zeta}(f, A) \leqslant e^{\varepsilon\left(r+\zeta^{\gamma}\right)}\left(r^{1 / \gamma}\left(a_{\gamma}+\delta\right)^{1 / \gamma} / \zeta\right)^{r}, \quad \zeta \geqslant \zeta_{\varepsilon}, r \geqslant r_{\delta} .
$$

Для произвольного $\xi \geqslant r_{\delta}$ выберем такое натуральное число $r$, что $r \leqslant \xi<$ $r+1$. Тогда для $\zeta \geqslant \zeta_{\varepsilon}$ и $\xi \geqslant r_{\delta}$ имеем

$$
\begin{aligned}
E_{\zeta}(f, A) & \leqslant e^{\varepsilon\left(\xi+\zeta^{\gamma}\right)}\left(\xi^{1 / \gamma}\left(a_{\gamma}+\delta\right)^{1 / \gamma} / \zeta\right)^{r} \\
& \leqslant e^{\varepsilon\left(\xi+\zeta^{\gamma}\right)} \max \left\{1, \zeta \xi^{-1 / \gamma}\left(a_{\gamma}+\delta\right)^{-1 / \gamma}\right\}\left(\xi^{1 / \gamma}\left(a_{\gamma}+\delta\right)^{1 / \gamma} / \zeta\right)^{\xi} .
\end{aligned}
$$

Считая, что $\zeta \geqslant \zeta_{\delta, \varepsilon}:=\max \left\{\zeta_{\varepsilon},\left(e r_{\delta}\left(a_{\gamma}+\delta\right)\right)^{1 / \gamma}\right\}$, и полагая в предыдущем неравенстве $\xi=e^{-1} \zeta^{\gamma}\left(a_{\gamma}+\delta\right)^{-1}$, получаем

$$
\begin{aligned}
E_{\zeta}(f, A) & \leqslant \exp \left\{\varepsilon\left(1+\frac{1}{e\left(a_{\gamma}+\delta\right)}\right) \zeta^{\gamma}+\frac{1}{\gamma}-\frac{\zeta^{\gamma}}{e \gamma\left(a_{\gamma}+\delta\right)}\right\} \\
& =\exp \left\{\frac{1}{\gamma}-\frac{1-\varepsilon \gamma\left(1+e\left(a_{\gamma}+\delta\right)\right)}{e \gamma\left(a_{\gamma}+\delta\right)} \zeta^{\gamma}\right\}, \quad \zeta \geqslant \zeta_{\delta, \varepsilon} .
\end{aligned}
$$

Отсюда и из соотношений (3.4) следует, что $E_{\zeta}(f, A) \rightarrow 0$ при $\zeta \rightarrow \infty$, т.е. $f \in \overline{\mathfrak{G}_{\infty}(A)}$, а учитывая определение (3.2) числа $b_{\gamma}$, выводим такую оценку:

$$
b_{\gamma} \leqslant \frac{e \gamma\left(a_{\gamma}+\delta\right)}{1-\varepsilon \gamma\left(1+e\left(a_{\gamma}+\delta\right)\right)} .
$$

Тем самым показано, что из включения $f \in \mathfrak{D}_{\infty}(A)$ и конечности $a_{\gamma}$ следует включение $f \in \overline{\mathfrak{G}_{\infty}(A)}$ и конечность $b_{\gamma}$. Кроме того, в неравенстве $(3.5) \varepsilon$ - произвольное число, удовлетворяющее неравенствам (3.4), а значит, (3.5) справедливо при $\varepsilon=0$. В свою очередь, $\delta$ - произвольное положительное число, и поэтому (3.5) справедливо при $\delta=0$, т. е. $b_{\gamma} \leqslant e \gamma a_{\gamma}$. 
Пусть теперь $f \in \overline{\mathfrak{G}_{\infty}(A)}$ и $b_{\gamma}$ конечно. Тогда $E_{\zeta}(f, A) \rightarrow 0$ при $\zeta \rightarrow \infty$ и для каждого $\delta>0$ найдется такое положительное $\zeta_{\delta}^{\prime}$, что

$$
E_{\zeta}(f, A)<1 \quad \text { и } \quad \frac{\zeta^{\gamma}}{-\ln E_{\zeta}(f, A)} \leqslant b_{\gamma}+\delta, \quad \zeta \geqslant \zeta_{\delta}^{\prime},
$$

т. е. $\ln E_{\zeta}(f, A) \leqslant-\zeta^{\gamma} /\left(b_{\gamma}+\delta\right), \zeta \geqslant \zeta_{\delta}^{\prime}$. Таким образом,

$$
E_{\zeta}(f, A) \leqslant c_{\delta}^{\prime} e^{-\zeta^{\gamma} /\left(b_{\gamma}+\delta\right)}, \quad \zeta \geqslant 0,
$$

где постоянная $c_{\delta}^{\prime}$ больше 0. Следовательно, условие (2.5) леммы 2.1 выполнено при любом $\varepsilon$, удовлетворяющем неравенствам

$$
0<\varepsilon<2^{-1}\left(b_{\gamma}+\delta\right)^{-1}
$$

Значит, $f \in \mathfrak{D}_{\infty}(A)$ и справедлива оценка (2.6). Полагая

$$
\nu:=\left(b_{\gamma}+\delta\right)^{-1}-\varepsilon,
$$

из оценок (2.6) и (3.6) получаем

$$
\left\|A^{r} f\right\| \leqslant 2 c_{\varepsilon} e^{\varepsilon r} \max \left\{\eta_{\varepsilon}^{r}\|f\|, c_{\delta}^{\prime} \int_{0}^{\infty} e^{-\nu \xi^{\gamma}} \xi^{r-1} d \xi\right\}, \quad r \in \mathbb{N} .
$$

Справедливо равенство

$$
\int_{0}^{\infty} e^{-\nu \xi^{\gamma}} \xi^{r-1} d \xi=\frac{1}{\gamma \nu^{r / \gamma}} \Gamma\left(\frac{r}{\gamma}\right)
$$

a, согласно формуле Стирлинга для гамма-функции, $\lim _{\zeta \rightarrow \infty} \zeta^{-1} \Gamma(\zeta)^{1 / \zeta}=e^{-1}$. Отсюда и из (3.1), (3.8) и (3.9) получаем

$$
a_{\gamma} \leqslant e^{\varepsilon \gamma} \frac{1}{e \gamma \nu}=e^{\varepsilon \gamma} \frac{b_{\gamma}+\delta}{e \gamma\left(1-\varepsilon\left(b_{\gamma}+\delta\right)\right)} .
$$

Здесь $\delta$ - произвольное положительное число, а $\varepsilon$ удовлетворяет неравенствам (3.7). Поэтому соотношения (3.10) справедливы при $\delta=\varepsilon=0$, т.е. $e \gamma a_{\gamma} \leqslant b_{\gamma}$. Значит, из включения $f \in \overline{\mathfrak{G}_{\infty}(A)}$ и конечности $b_{\gamma}$ следует включение $f \in \mathfrak{D}_{\infty}(A)$ и конечность $a_{\gamma}$, а ввиду ранее доказанного неравенства $b_{\gamma} \leqslant e \gamma a_{\gamma}$ получаем равенство $е \gamma a_{\gamma}=b_{\gamma}$.

ВЫвод теОремы 1.1. Учитывая формулу (1.3) для вычисления $R_{A}(f)$ и определение (3.1) числа $a_{\gamma}$, имеем $R_{A}(f)=\left(e a_{1}\right)^{-1}$. Поэтому $R_{A}(f)>0$ в том и только том случае, когда $a_{1}<\infty$. Отсюда и из теоремы 3.1 при $\gamma=1$ получается теорема 1.1.

Некоторым усилением теоремы 1.2 является следующее утверждение, в формулировке которого $\sigma_{A}^{(1)}(f)$ и $\sigma_{E}(f, A)$ заданы соответственно равенствами (1.9) при $\rho=1$ и (1.10), причем если вектор $f$ не принадлежит $\mathfrak{D}_{\infty}(A)$, то считаем, что $\sigma_{A}^{(1)}(f):=\infty$.

ТеОРема 3.2. Справедливо неравенство

$$
\sigma_{E}(f, A) \leqslant \sigma_{A}^{(1)}(f) .
$$

Если $A \in \mathscr{R}_{\gamma}$ при некотором $\gamma>0$, то для каждого $\varepsilon>0$ найдется такое $\eta_{\varepsilon}>0$ (то же самое, ито и в лемме 2.1), для которого

$$
\sigma_{A}^{(1)}(f) \leqslant e^{\varepsilon} \max \left\{\sigma_{E}(f, A), \eta_{\varepsilon}\right\} .
$$


ДоказАтЕльство. Неравенства (3.11) и (3.12) содержательны соответственно в случаях, когда $\sigma_{A}^{(1)}(f)<\infty$ и $\sigma_{E}(f, A)<\infty$; поэтому при их доказательстве будем придерживаться этих предположений.

Из определения $\sigma_{A}^{(1)}(f)$ следует, что для произвольного $\delta>0$ найдется такая постоянная $c(\delta, f)>0$, для которой $\left\|A^{r} f\right\| \leqslant c(\delta, f)\left(\sigma_{A}^{(1)}(f)+\delta\right)^{r}, r \in \mathbb{P}$, т. е. $f \in \mathfrak{G}_{\xi}(A)$ при $\xi=\sigma_{A}^{(1)}(f)+\delta$, а значит, $E_{\zeta}(f, A)=0$ при $\zeta \geqslant \sigma_{A}^{(1)}(f)+\delta$. Отсюда в силу произвольности $\delta>0$ получается оценка (3.11).

Из определения числа $\sigma_{E}(f, A)$ и из предположения о его конечности заключаем, что требование (2.5) выполнено при произвольном $\varepsilon>0$. Поэтому на основании второго утверждения леммы 2.1 вектор $f$ принадлежит $\mathfrak{D}_{\infty}(A)$ и справедлива оценка (2.6) с заменой в интеграле из ее правой части верхнего предела интегрирования, равного $\infty$, на $\sigma:=\sigma_{E}(f, A)$. Отсюда и из неравенства $E_{\xi}(f, A) \leqslant\|f\|$ имеем

$$
\left\|A^{r} f\right\| \leqslant c_{\varepsilon} e^{\varepsilon r}\|f\|\left(\eta_{\varepsilon}^{r}+\int_{0}^{\sigma} e^{\varepsilon \xi^{\gamma}} \xi^{r-1} d \xi\right) \leqslant 2 c_{\varepsilon} e^{\varepsilon\left(r+\sigma^{\gamma}\right)}\|f\|\left(\max \left\{\sigma, \eta_{\varepsilon}\right\}\right)^{r} .
$$

Учитывая, что в этих оценках $\sigma=\sigma_{E}(f, A)$, из них и из определения (1.9) при $\rho=1$ числа $\sigma_{A}^{(1)}(f)$ получаем (3.12).

Теорема 1.2 является очевидным следствием теоремы 3.2 .

Из теоремы 3.1 просто выводится

СледСТвиЕ 3.1. Пусть $A \in \mathscr{R}_{\gamma}$ при некотором $\gamma>0 u \sigma_{E}(f, A)>0$. Тогда для того чтобы вектор $f$ принадлежал $\mathfrak{D}_{\infty}(A)$ и число

$$
\alpha_{A}(f):=\varlimsup_{r \rightarrow \infty} \frac{\ln \left\|A^{r} f\right\|}{r \ln r}
$$

удовлетворлло неравенству $\alpha_{A}(f)<\gamma^{-1}$, необходимо и достаточно, итобь $f$ принадлежал $\overline{\mathfrak{G}_{\infty}(A)}$ и число $\beta_{A}(f)$, заданное формулой (1.11), удовлетворяло неравенству $\beta_{A}(f)<\gamma^{-1}$. При этом $\alpha_{A}(f)=\beta_{A}(f)$.

Отметим лишь, что требование $\sigma_{E}(f, A)>0$ гарантирует неотрицательность $\alpha_{A}(f)$. Действительно, если $\alpha_{A}(f)<0$, то $\sigma_{A}^{(1)}(f)=0$, а отсюда и из (3.11) имеем $\sigma_{E}(f, A)=0$.

Вывод теоремы 1.3. Теорема 1.3 вытекает из следствия 3.1, формул (1.8), (1.9) и теоремы 3.1. При этом следует лишь заметить, что так как в теореме $1.3 \gamma \geqslant 1$, то $\beta_{A}(f)<\gamma^{-1} \leqslant 1$ и поэтому, согласно следствию $3.1, \alpha_{A}(f)<1$. Отсюда и из формул (1.3) и (3.13) легко заключить, что $f$ - целый вектор оператора $A$.

Как видно из вывода теорем 1.1 и 1.3 из теоремы 3.1, эта теорема является уточнением предыдущих на случай неголоморфных векторов. Если в теореме 3.1 положить $A=D$, где $D$ задан соотношениями (1.7), то из нее просто выводится один из результатов С. Н. Бернштейна [1, работа 89, теорема 3$]$.

Вывод теоремы 1.4. Неравенство $\sigma_{E}(f, A) \leqslant \sigma_{A}^{(1)}(f)$ установлено в теореме 3.2 без каких-либо ограничений относительно $A$. Поэтому для доказательства теоремы 1.4 достаточно показать, что если оператор $A$ имеет мероморфную резольвенту, то $\sigma_{A}^{(1)}(f) \leqslant \sigma_{E}(f, A)$. Пусть $\zeta>\sigma_{E}(f, A)$, т. е. $E_{\zeta}(f, A)=0$. Согласно предложению $1.1, \mathfrak{G}_{\zeta}(A)$ замкнуто, а значит, $f \in \mathfrak{G}_{\zeta}(A)$ и найдутся 
такие цепочки $y_{0, k}, \ldots, y_{k, d_{k}}$ собственных и присоединенных к ним векторов, отвечающие собственным значениям $\lambda_{k}(A)$, что $f=\sum_{k:\left|\lambda_{k}(A)\right| \leqslant \zeta} y_{k, d_{k}}$, и поэтому

$$
e^{z A} f=\sum_{k:\left|\lambda_{k}(A)\right| \leqslant \zeta} e^{z \lambda_{k}(A)} \sum_{s=0}^{d_{k}} \frac{z^{s}}{s !} y_{k, d_{k}-s} .
$$

Следовательно, тип функции $e^{z A} f$ не выше $\zeta$. Но $\zeta-$ произвольное число, большее $\sigma_{E}(f, A)$, а значит, тип функции $e^{z A} f$ не выше $\sigma_{E}(f, A)$.

Если нуль является собственным значением оператора $A$ и $\sigma_{E}(f, A)=0$, то, полагая в равенстве (3.14) положительное $\zeta$ столь малым, чтобы в круге $\{\lambda \in \mathbb{C}:|\lambda| \leqslant \zeta\}$ имелось лишь одно нулевое собственное значение, из (3.14) получаем последнее утверждение теоремы 1.4 .

\section{ЛИТЕРАТУРА}

1. Бернштейн C. Н. Собрание сочинений, Т. II. Изд-во АН СССР, М., 1954.

2. Nelson E. Analytic vectors. Ann. of Math. (2), 70, 572-615 (1959).

3. Хилле Э., Филлипс Р. Функциональный анализ и полугруппы. ИЛ, М., 1962.

4. Радзиевский Г. В. О наилучших приближениях и о скорости сходимости разложений по корневым векторам оператора. Укр. матем. ж., 49, №6, 754-773 (1997).

5. Goodman $R$. Analytic and entire vectors for representations of Lie groups. Trans. Amer. Math. Soc., 143, 55-76 (1969).

6. Леонтьев А. Ф. Целые функции. Ряды экспонент. Наука, М., 1983.

7. Леонтьев А. Ф. Последовательности полиномов из экспонент. Наука, М., 1980.

8. Радзиевский Г. В. Асимптотика собственных значений регулярной краевой задачи. Укр. матем. ж., 48, № 4, 483-519 (1996).

9. Данфорд Н., Швари Дж. Т. Линейные операторы. Общая теория. ИЛ., М., 1962.

Институт математики НАНУ,

Киев, Украина
Поступило в редакцию 6 августа 1997 г. 